\title{
Evaluating New Varieties of Wheat with the Application of Vague Optimization Methods
}

\author{
Hongxu Wang ${ }^{1}$, FuJin Zhang ${ }^{2}$, and Yunsheng $\mathrm{Xu}^{3}$ \\ ${ }^{1,3}$ College of Science and Engineering, \\ ${ }^{2}$ College of Electronic Information Engineering, Qiongzhou University, \\ Sanya Hainan 572022, China \\ zfj56801@163.com, whx16233@yahoo.cn
}

\begin{abstract}
The Vague optimization method sorted out is a special case of the pattern recognition method of the Vague set. Its specific application steps are as follows: 1 the set-up of characters to determine the evaluation; 2 the establishment of a collection of new varieties of wheat to be optimized; 3 the extract of the ideal set of new wheat varieties ; 4 the construction of Vague environment to collect all varieties of Vague sets; 5 the Vague optimization: to calculate similarity measures between Vague sets to obtain the new wheat varieties based on numerical similarity measures and to propose the similarity measures formula between the Vague sets. This optimization formula is supported by Vague class techniques. Breeding new varieties of wheat with comprehensive quality traits is one of the directions in wheat breeding. It is a new attempt to study the wheat assessment of the new wheat varieties with the application of optimization methods. The wheat assessment case of the new wheat varieties shows that both the formula and the method are practical.
\end{abstract}

Keywords: Vague set, Vague optimization, Vague environment, similarity measure, assessment of new varieties of wheat.

\section{Introduction}

Vague sets [1] were put forward as Fuzzy Sets' population[1]. Though it was put forward 28 years later than Fuzzy set theory, Vague set theory has been widely used in many fields such as in pattern recognition, automatic control, intelligent reasoning, for it uses the interval numbers of Vague set membership to indicate degree of membership, which can more fully reflect the fuzzy information. This paper aims to apply the Vague set theory to the evaluation of new varieties of wheat and to provide the new research methods to the agriculture-related issues.

\section{Basic Concept}

Definition 1[1]. Suppose $L$ be a point of space, any one of the elements can be indicated with $l$. $L$ on a Vague set is $\mathrm{S}$, it is a membership function with a true $t_{s}$ and 
a false membership function $f_{S}$ said. $t_{S}(l) l$ is evidence to support the export of certain lower bound of the membership, and $l$ is the evidence from the opposition $f_{S}(l)$ derived the lower bound of the negative membership. $t_{S}(l)$ and $f_{S}(l)$ will be a real number in the range of point of contact with the $L$ together. That is, $\operatorname{map} t_{S}: L \rightarrow[0,1], f_{S}: L \rightarrow[0,1]$, and satisfies the constraints: $1 \geq t_{S}(l)+f_{S}(l)$.Vague set can be recorded $L$ points $l$ in the membership or Vague is simplified by $S(l)=\left[t_{S}(l), 1-f_{S}(l)\right]$ or $l=\left[t_{l}, 1-f_{l}\right]$.

It can be seen from the definition of $l=\left[t_{l}, 1-f_{l}\right] \subseteq[0,1] . \pi_{S}=1-t_{S}-f_{S}$ that on the set of $S$ elements in $l$ pairs is uncertain function Vague, also called the degree of uncertainty or the degree of Vague. If $L$ is a discrete domain of $L=\left\{l_{1}, l_{2}, \cdots, l_{n}\right\}$ and $L$ on Vague set $S$ can be written as $S=\sum_{i=1}^{n} \frac{\left[t_{S}\left(l_{i}\right), 1-f_{S}\left(l_{i}\right)\right]}{l_{i}}$, also denoted by $S=\sum_{i=1}^{n} \frac{\left[t_{l_{i}}, 1-f_{l_{i}}\right]}{l_{i}}$.

\section{Vague Environmental Construction}

The so-called construction of Vague environment is to turn the raw data into Vague data. This step is a prerequisite for the application of Vague sets. The single-value data are turned into the data definition Vague proposed in Reference [3], here again the formula for turning single-value data into a Vague data is provided in the assessment of new wheat varieties.

Definition 2. Let the domain $L=\left\{l_{1}, l_{2}, \cdots, l_{n}\right\}, L$, has a collection of $S_{i}(i=1,2, \cdots m), S_{i} l_{j}(j=1,2, \cdots n)$ data set of characters with non-negative singlevalue data $l_{i j}$. Suppose when the single-value data when the non-negative $l_{i j}$ Vague data turning into the formula to meet the output of the conversion of $S_{i}\left(l_{j}\right)=l_{i j}=\left[t_{i j}, 1-f_{i j}\right]$ conditions and Vague terms, we call this formula the formula for the output type, among which:

\section{a. Output Conditions}

If $0 \leq l_{k j}<l_{i j}, l_{i j}$ and $l_{k j}$ single-value data turn into the Vague data respectively, $S_{i}\left(l_{j}\right)=l_{i j}=\left[t_{i j}, 1-f_{i j}\right] \quad$ and $\quad S_{k}\left(l_{j}\right)=l_{k j}=\left[t_{k j}, 1-f_{k j}\right] \quad$ satisfy the condition: $t_{k j} \leq t_{i j}, 1-f_{k j} \leq 1-f_{i j}$.

\section{b. Input Conditions}

If $0 \leq l_{k j}<l_{i j}, l_{i j}$ and $l_{k j}$ single-value data turn into the Vague data, $S_{i}\left(l_{j}\right)=l_{i j}=\left[t_{i j}, 1-f_{i j}\right] \quad$ and $\quad S_{k}\left(l_{j}\right)=l_{k j}=\left[t_{k j}, 1-f_{k j}\right] \quad$ satisfy the condition: $t_{k j} \geq t_{i j}, 1-f_{k j} \geq 1-f_{i j}$ 
c. Vague Terms $\quad 0 \leq t_{i j} \leq 1-f_{i j} \leq 1$.

Note: Output type transformation formula for numerical characters takes "the bigger, the better" in use; input type transformation formula for numerical characters takes "the smaller the better" in use.

Theorem 1: If $l_{j \max }=\max \left\{l_{1 j}, l_{2 j}, \cdots, l_{m j}\right\}$, then

$$
S_{i}\left(l_{j}\right)=l_{i j}=\left[t_{i j}, 1-f_{i j}\right]=\left[\left(\frac{l_{i j}}{l_{j \max }}\right)^{2},\left(\frac{l_{i j}}{l_{j \max }}\right)^{\frac{1}{2}}\right]
$$

is non-negative single-value data $l_{i j}$ into the output data type Vague conversion formula.

$$
S_{i}\left(l_{j}\right)=l_{i j}=\left[t_{i j}, 1-f_{i j}\right]=\left[1-\left(\frac{l_{i j}}{l_{j \max }}\right)^{\frac{1}{2}}, 1-\left(\frac{l_{i j}}{l_{j \max }}\right)^{2}\right]
$$

is non-negative single-value data $l_{i j}$ into the input data type Vague conversion formula.

\section{The New Similarity Measures between Vague Values}

If Lemma [4] is to Vague value of $l=\left[t_{l}, 1-f_{l}\right]$, then $l^{(m)}=\left[t_{l}^{(m)}, 1-f_{l}^{(m)}\right]$ is Vague value. Vague value $l=\left[t_{l}, 1-f_{l}\right]$ of the formula is known as the first $m$ to $\left(t_{l}, f_{l}\right)$ data mining Vague value, among which:

$$
\begin{aligned}
& f_{l}^{(m)}=f_{l}\left(1+\pi_{l}+\pi_{l}^{2}+\cdots+\pi_{l}^{m}\right), \alpha_{l}^{(m)} \\
& =\left(t_{l}+f_{l}\right)\left(1+\pi_{l}+\pi_{l}^{2}+\cdots+\pi_{l}^{m}\right), \beta_{l}^{(m)} \\
& =\left(t_{l}-f_{l}\right)\left(1+\pi_{l}+\pi_{l}^{2}+\cdots+\pi_{l}^{(m)}\right),(m=0,1,2, \cdots) .
\end{aligned}
$$

A simple and practical Vague measure of similarity between the values is defined by Reference [5].

Definition 3. If $s=\left[t_{s}, 1-f_{s}\right], h=\left[t_{h}, 1-f_{h}\right]$ is two Vague values, the formula $M(s, h)$ between $s$ and $h$ is similarity measures of the Vague value, if $M(s, h)$ satisfies the following conditions:
a. $0-1$ conditions
$0 \leq M(s, h) \leq 1$
b. Symmetry conditions $M(s, h)=M(h, s)$
c. Reflexive conditions $M(s, s)=1$
d. Minimum condition 
When $s=[0,0], h=[1,1]$ or $s=[1,1], h=[0,0]$, the $M(s, h)=0$. Vague, which is also known as the numerical value of $M(s, h)=0$ similarity between $s$ and $h$.

Note: the similarity measure of Vague value between $s$ and $h$, means that the greater the similarity values of $M(s, h), \mathrm{s}$ and $\mathrm{h}$, the more similar Vague values between $s$ and $h$, especially when the maximum similarity value of $M(s, h)$ is up to 1 , the value of Vague $s$ and $h$ is the most similar; conversely, the smaller the similarity value of $M(s, h), s$ and $h$, less similar Vague values between $s$ and $h$, especially when the minimum similarity value of $M(s, h)$ reaches 0 , value of Vague $s$ and $h$ is the least similar.

Theorem 2. Note $m=0,1,2, \cdots$, the formula

$$
M_{m}(s, h)=\left[1-\left|f_{s}^{(m)}-f_{h}^{(m)}\right|\right] \cdot\left[1-\frac{\left|\beta_{s}^{(m)}-\beta_{h}^{(m)}\right|+\left|\alpha_{s}^{(m)}-\alpha_{h}^{(m)}\right|}{2}\right]
$$

is the similarity measure of Vague value between $s$ and $h$.

\section{$5 \quad$ Vague Similarity Measures between the New Vague Sets of Metrics}

From the definition of similarity measures between Vague Sets and Vague Sets similarity between the weighted measure, similar to Definition 3, theorem 2 can also be obtained from the following theorem.

Theorem 3. Let the domain $L=\left\{l_{1}, l_{2}, \cdots, l_{n}\right\}, L$ on $S=\sum_{i=1}^{n} \frac{\left[t_{S}\left(l_{i}\right), 1-f_{S}\left(l_{i}\right)\right]}{l_{i}}$ and $H=\sum_{i=1}^{n} \frac{\left[t_{H}\left(l_{i}\right), 1-f_{H}\left(l_{i}\right)\right]}{l_{i}}$ Vague sets are simply denoted as $S=\sum_{i=1}^{n} \frac{\left[t_{s_{i}}, 1-f_{s_{i}}\right]}{l_{i}}$ and $H=\sum_{i=1}^{n} \frac{\left[t_{h_{i}}, 1-f_{h_{i}}\right]}{l_{i}}$ respectively. And note $m=0,1,2, \cdots$, The formula

$$
M_{m}(S, H)=\frac{1}{n} \sum_{i=1}^{n}\left[1-\left|f_{s_{i}}^{(m)}-f_{h_{i}}^{(m)}\right|\right] \cdot\left[1-\frac{\left|\beta_{s_{i}}^{(m)}-\beta_{h_{i}}^{(m)}\right|+\left|\alpha_{s_{i}}^{(m)}-\alpha_{h_{i}}^{(m)}\right|}{2}\right]
$$

is the similarity measures of Vague sets between $s$ and $h$ of the metrics.

Theorem 4. If the element weight $l_{i}(i=1,2, \cdots, n)$ is $b_{i} \in[0,1]$, and $\sum_{i=1}^{n} b_{i}=1$, and note $m=0,1,2, \cdots$. then in the conditions in Theorem 3 , the formula

$$
M_{m}(S, H)=\sum_{i=1}^{n} b_{i} \cdot\left[1-\left|f_{s_{i}}^{(m)}-f_{h_{i}}^{(m)}\right|\right] \cdot\left[1-\frac{\left|\beta_{s_{i}}^{(m)}-\beta_{h_{i}}^{(m)}\right|+\left|\alpha_{s_{i}}^{(m)}-\alpha_{h_{i}}^{(m)}\right|}{2}\right]
$$

is the weighted similarity measures between Vague sets $S$ and $H$. 


\section{$6 \quad$ Vague Optimization}

The comprehensive decision-making rules of Vague sets in Reference [6] are reorganized into Vague optimization method fit for the research on the assessment of new varieties of wheat. Their specific application steps are as follows: 1 the set-up of characters to determine the evaluation; 2 the establishment of a collection of new varieties of wheat to be optimized; 3 the extract of the ideal set of new wheat varieties; 4 the construction of Vague environment to collect all varieties of Vague sets; 5 the Vague optimization: to calculate similarity measures between Vague sets to obtain the new wheat varieties based on numerical similarity measures. If Vague sets of pattern recognition is for the purpose of optimization, then the pattern recognition method of Vague sets can be called the Vague optimization method. So Vague set optimization method is a special case of the pattern recognition. It has one standard model and several to be recognized over the Vague sets of pattern recognition methods.

\section{Optimal Assessment of New Varieties of Wheat}

Fuzzy Comprehensive Evaluation in Reference [7] is used to study the assessment of new varieties of wheat. In this paper, Vague optimization method is applied to re-examine the issue.

\subsection{To Establish the Evaluation Set of Traits}

Take $l_{1}$ as the "yield $(\mathrm{kg} / 667 \mathrm{~m}) " ; l_{2}$ as "strain rate $(\%) " ; l_{3}$ as the" drought index "; $l_{4}$ as a" valid spike number (million / 667m) "; $l_{5}$ as" grains per spike ( tablets) "; $l_{6}$ as" grain weight $(\mathrm{g})$ ", then the evaluation of the set of characters is $L=\left\{l_{1}, l_{2}, \cdots, l_{6}\right\}$.

Table 1. The characteristics of new varieties of wheat, the average data

\begin{tabular}{cccccc}
\hline Factor & \multicolumn{6}{c}{ The characteristics of new varieties of wheat, the average data } \\
\hline & $S_{1}$ & $S_{2}$ & $S_{3}$ & $S_{4}$ & $H$ \\
\hline$l_{1}$ & 285.8 & 278.1 & 271.3 & 256.7 & 285.8 \\
\hline$l_{2}$ & 6.4 & 3.3 & 10.3 & 7.8 & 3.3 \\
\hline$l_{3}$ & 1.203 & 1.413 & 1.039 & 1.101 & 1.413 \\
\hline$l_{4}$ & 38.80 & 40.45 & 34.5 & 35.60 & 40.45 \\
\hline$l_{5}$ & 33.1 & 32.3 & 32.4 & 32.0 & 33.1 \\
\hline$l_{6}$ & 37.7 & 32.5 & 38.8 & 37.4 & 38.8 \\
\hline
\end{tabular}




\subsection{To Set Up the Optimal Set of New Varieties of Wheat}

Take $S_{1}$ as the "Liao $97 \mathrm{Kam} 30 ", S_{2}$ as "Zhemai 10", $S_{3}$ as "Kyrgyzstan Spring 9806", $S_{4}$ as the "Liao Chun 9 (ck)". They are composed of a collection of traits evaluated on a set of $L=\left\{l_{1}, l_{2}, \cdots, l_{6}\right\}$. They consist of a collection of new varieties of wheat to be optimized. The specific data are from Reference [7], as shown in Table 1.

\subsection{To Extract the Ideal Project}

Reference [7] tells that if the new varieties of wheat are expected to be all "optimal" in all traits, then the bigger the $l_{1}$ is, the better should be; the smaller the $l_{2}$ is, the better; the bigger the $l_{3}$ is, the better; the more the $l_{4}$ is, the better; the more the $l_{5}$ is the better; the heavier $l_{6}$ is, the better. Extracting the best data of various traits available to evaluate the set of characters composed of a collection of $L=\left\{l_{1}, l_{2}, \cdots, l_{6}\right\}$. on the $H$, which is called the ideal of new wheat varieties. The data for each trait are shown in Table 1.

\subsection{Enter the Vague Environment}

Formula (1) can be applied to Characters in $l_{1}, l_{3}, l_{4}, l_{5}$ and $l_{6}$; Formula (2) is applied to $l_{2}$. Table 1 can be turned into Table 2 .

Table 2. The characteristics of new varieties of wheat Vague data

\begin{tabular}{ccccccc}
\hline Factor & \multicolumn{5}{c}{ The characteristics of new varieties of wheat } & Vague data \\
\hline & $S_{1}$ & $S_{2}$ & $S_{3}$ & $S_{4}$ & $H$ \\
\hline$l_{1}$ & {$[1.000,1.000]$} & {$[0.947,0.986]$} & {$[0.901,0.974]$} & {$[0.806,0.948]$} & {$[1.000,1.000]$} \\
\hline$l_{2}$ & {$[0.212,0.614]$} & {$[0.434,0.898]$} & {$[0.000,0.000]$} & {$[0.271,0.438]$} & {$[0.434,0.898]$} \\
\hline$l_{3}$ & {$[0.724,0.922]$} & {$[1.000,1.000]$} & {$[0.540,0.857]$} & {$[0.607,0.880]$} & {$[1.000,1.000]$} \\
\hline$l_{4}$ & {$[0.920,0.979]$} & {$[1.000,1.000]$} & {$[0.728,0.924]$} & {$[0.774,0.938]$} & {$[1.000,1.000]$} \\
\hline$l_{5}$ & {$[1.000,1.000]$} & {$[0.953,0.988]$} & {$[0.958,0.989]$} & {$[0978,0.994]$} & {$[1.000,1.000]$} \\
\hline$l_{6}$ & {$[0.945,0.986]$} & {$[0.702,0.915]$} & {$[1.000,1.000]$} & {$[0.929,0.982]$} & {$[1.000,1.000]$} \\
\hline
\end{tabular}

Table 2 shows the selection of the new wheat varieties of $S_{1}, S_{2}, S_{3}, S_{4}$, and $H$ new varieties of wheat ideal Vague collection.

\subsection{Vague Optimization}

New varieties of wheat are sorted out through calculating the similarity measures between Vague sets, according to the size of numerical similarity measure. The 
formula (4) is as follows: take $m=2$, calculate the similarity measures between Vague sets $S_{i}(i=1,2,3,4)$ and $H$. The results are:

$$
M_{2}\left(S_{1}, H\right)=0.843, M_{2}\left(S_{2}, H\right)=0.956, M_{2}\left(S_{3}, H\right)=0.729, M_{2}\left(S_{4}, H\right)=0.770 .
$$

Thus, according to the size of similarity measures, the order is:

$$
M_{2}\left(S_{2}, H\right)>M_{2}\left(S_{1}, H\right)>M_{2}\left(S_{4}, H\right)>M_{2}\left(S_{3}, H\right) .
$$

The similarity of Vague sets is that the larger the number is, the more similar these two Vague sets are; the smaller the value is, the more dissimilar the two Vague sets are. So the conclusion of Vague optimization decision: among which the best variety is $S_{2}$ : Zhemai 10 . The second is $S_{1}$ : Liao $97 \mathrm{Kam} \mathrm{30.} \mathrm{and} \mathrm{poorer} \mathrm{one} \mathrm{is} S_{4}$ : Liao Chun 9 (ck) and $S_{3}$ : Ji Chun 9806, $S_{4}$ : Liao Chun 9 (ck).

\section{Conclusion}

The Fuzzy comprehensive evaluation method used applied in Reference [7], although it is able to "make up for the lack of analysis of variance," is relatively more complicated, in particular it needs to calculate fuzzy matrix, whose speed is rather slow. The optimization method, by means of wheat Vague Selection of new varieties, is feasible. It is more reasonable and easier to use than the existing one, and it provides a more practical approach in optimizing the new wheat varieties. tend to provide. From the example mentioned-above, Vague optimization method is an alternative to the fuzzy comprehensive evaluation. As we all know, the application of fuzzy comprehensive evaluation process is more cumbersome, and with a large number of calculation. Vague optimization method not only provides a new method for discussing the similar problems, and its application in the fields such as species optimization is of a great potential area. And the formula (1), (2), (3), (4) and (5) put forward in this paper provide the technical support for it.

Acknowledgments. The Hainan Provincial Natural Science Fund Project No.610224; the Hainan Provincial Social development projects for science and technology development fund No.2010SF004 and Sanya City College, 2009 special fund project funding issues (YD09027).

\section{References}

1. Gau, W.-L., Buehrer, D.J.: Vague Sets. IEEE Transactions on Systems, Man and Cybernetics 23(2), 610-614 (1993)

2. Zadeh, L.A.: Fuzzy Sets. Information and Control (8), 338-353 (1965)

3. Wang, H.-x.: Definition and transforming formulas from the single valued data to the vague valued data. Computer Engineering and Applications 46(24), 42-44 (2010)

4. Liu, H.-W., Wang, F.-Y.: Transformations and Similarity Measures of Vague Sets. Computer Engineering and Applications 40(32), 79-81 (2004) 
5. Wang, H.-X.: Similarity measure between vague sets and their application. Computer Engineering and Applications 46(26), 198-199 (2010)

6. Wang, H.-X.: Synthesis decision rule of vague sets and its application in scheme optimum seeking. Computer Engineering and Applications 46(27), 145-147 (2010)

7. Modeleto, Erdunga, Bayilatu, et al.: The fuzzy comprehensive evaluation to the application of new wheat evaluation. Inner Mongolia Agricultural Science and Technology (1), 37-38 (2007) 